\title{
Asymptotic Analysis of Risk Quantities Conditional on Ruin For Multidimensional Heavy-tailed Random Walks
}

\author{
Jingchen Liu* $^{*}$ and Jae-Kyung Woo ${ }^{\dagger}$
}

August 29, 2013

\begin{abstract}
In this paper we consider a multidimensional renewal risk model with regularly varying claims. This model may be used to describe the surplus of an insurance company possessing several lines of business where a large claim possibly puts multiple lines in a risky condition. Conditional on the occurrence of ruin, we develop asymptotic approximations for the average accumulated number of claims leading the process to a rare set, and the expected total amount of shortfalls to this set in finite and infinite horizons. Furthermore, for the continuous time case, asymptotic results regarding the total occupation time of the process in a rare set and time-integrated amount of shortfalls to a rare set are obtained.
\end{abstract}

Keywords: multivariate regularly variation, heavy-tailed increments, hitting rare set, Lyapunov inequality.

\section{Introduction}

Multidimensional risk model is a useful tool to portray the overall solvency of insurance companies with multiple lines of business that may be affected by common shocks. A catastrophic event usually causes severe losses and results in several types of casualty insurance claims. For instance, flood causes claims of both automobiles and houses. In this case, multidimensional risk model attempts to capture the financial position of companies that can be significantly affected by risk events of such a grand scale.

To describe the tail dependence phenomena of large claims among different lines of business, we assume that the claim size follows a multivariate regularly varying distribution. This class of distributions is a popular choice to characterize dependence of extremal events. For instance, due to the tail dependence, if the claim size of one business line is huge, then there is a substantial probability that the claims of other lines are of comparable sizes. This phenomenon is often observed when the claims in different directions are caused by some common catastrophic events.

\footnotetext{
*Department of Statistics, Columbia University, 1255 Amsterdam Avenue, New York, NY, 10027, USA. Email address: jcliu@stat.columbia.edu

${ }^{\dagger}$ Department of Statistics and Actuarial Science, University of Hong Kong, Pokfulam Road, Hong Kong. Email address: jkwoo@hku.hk
} 
Let us start with a $d$-dimensional continuous time risk reserve vector process $\left\{U_{t}\right\}_{t \geq 0}$ representing the available reserve level of multiple business lines of an insurance company at time $t$. The claim arrivals follow a renewal process $\left\{N_{t}\right\}_{t \geq 0}$ with the interclaim times being a sequence of independent and identically distributed (i.i.d.) random variables $\left\{V_{i}\right\}_{i=1}^{\infty}$ with a general not necessarily exponential distribution, the generic random variable of which is denoted by $V$. The claim sizes, $\left\{Y_{i}\right\}_{i=1}^{\infty}$, independent of the interclaim times are a sequence of i.i.d. $\mathbb{R}^{d}$-valued regularly varying random vectors with index $\alpha$ denoted as $\operatorname{RV}(\alpha, \mu)$, where $\mu$ is the limiting measure of the distribution. A detail description of multivariate regularly varying distributions is provided in Section 2.1. The insurer's reserve process at time $t$ with initial reserve $R_{0} \in \mathbb{R}^{d}$ is given by

$$
U_{t}=R_{0}+c t-\sum_{i=1}^{N_{t}} Y_{i}, \quad t \geq 0
$$

where $c \in(0, \infty)^{d}$ is a deterministic vector representing the premium rates. We further define the associated claim surplus process

$$
W_{t}=\sum_{i=1}^{N_{t}} Y_{i}-c t, \quad t \geq 0
$$

In this paper, we concern events associated with ruin that occurs only at claim arrival times. It is sufficient to consider the reserve process at claim instances. Let us now consider a discrete time $d$-dimensional risk reserve vector process $\left\{R_{n}\right\}_{n=1}^{\infty}$ and a claim surplus process at the arrival of the $n$-th claim $\left\{S_{n}\right\}_{n=1}^{\infty}$ defined as

$$
R_{n}=U_{V_{1}+\ldots+V_{n}}, \quad S_{n}=W_{V_{1}+\ldots+V_{n}}, \quad n=1,2, \ldots
$$

Furthermore, the discrete processes adopt a random walk structure

$$
S_{n}=\sum_{i=1}^{n} X_{i}, \quad R_{n}=R_{0}-S_{n}=R_{0}-\sum_{i=1}^{n} X_{i}, \quad n=1,2, \ldots,
$$

where $\left\{X_{i}=Y_{i}-V_{i} c: i=1,2, \ldots\right\}$ is a sequence of i.i.d. increments. Let $X$ be the generic random vector equal in distribution to $X_{i}$. We further assume $E\left(V^{r}\right)<\infty$ for some $r>\alpha$. Then, the regular variation of $Y$, that is, $Y \in \operatorname{RV}(\alpha, \mu)$, implies that $X \in \operatorname{RV}(\alpha, \mu)$ (e.g. [28]). Therefore, $S_{n}$ is a discrete-time $d$-dimensional random walk with multivariate regularly varying increments.

We are primarily interested in the conditional distribution of the claim surplus process $S_{n}$ given the ruin event that $S_{n}$ falls into certain ruin set. This ruin set denoted by $A$ can be written as the union of finitely many half spaces of $\mathbb{R}^{d}$. Also, define the first passage time of $S_{n}$ to the ruin set

$$
\tau_{A}=\inf \left\{n \geq 0: S_{n} \in A\right\} .
$$

Similarly, for the continuous-time claim surplus process, we define stopping time as

$$
\xi_{A}=\inf \left\{t \geq 0: W_{t} \in A\right\}
$$


We will later choose the ruin set $A$ such that $\xi_{A}$ coincides the claim arrivals, that is,

$$
\xi_{A}=\sum_{i=1}^{\tau_{A}} V_{i} .
$$

implying that the visit of set $A$ is caused by claims and thus $\left\{\tau_{A}<\infty\right\}$ and $\left\{\xi_{A}<\infty\right\}$ are equivalent.

Our interest focuses on the asymptotic regime for which $A$ deviates from the origin in some opposite direction where the random walk drifts. This corresponds to the situation that the initial reserve of each line of business is large. More precisely, we multiply the set $A$ by a rarity parameter $b$. In practice, the parameter $b$ should be determined by the initial reserve vector $R_{0}$. Then, the ruin set is $b A=\{b \cdot x: x \in A\}$ and ruin occurs if $\tau_{b A}<\infty$. Our study focuses primarily on the condition measure $P\left(\cdot \mid \tau_{b A}<\infty\right)$ as $b \rightarrow \infty$. Regularity conditions will be imposed to ensure that $b A$ is a rare set. Indeed, in Section 2.2 conditions on the set $A$ are presented excluding the cases in which the probability of hitting a ruin set equals 1 (i.e. safety loading condition, see e.g. [1, p.3] and also [20] for the high dimensional setting). These notions will be given precisely in Section 2.1 and Section 2.2.

Under the one-dimensional setting, the first passage time of random walk is a classic problem in applied probability and it has been studied intensively in many areas such as queueing theory, branching processes, and dam/storage processes. However, there are much fewer works on exact results under the multidimensional setting in the context of risk theory. With a relatively simple risk model (i.e. compound Poisson risk model), for example, [19] considers a common shock model for a multidimensional risk process. Also, [3] studies a two-dimensional risk model where two lines of business are connected in terms of a quota share reinsurance treaty and additionally one of them has its own aggregate claim process, which is a generalization of [2]. Within the risk theory context, other studies concerning multidimensional problems are also given by [10, 8, 9, 29, 22, 2, 15, 27, 14]. Whereas most of these studies deal with the problems in two-dimensional compound Poisson model, the current work makes a generalization to the model with higher dimensions and more general claim arrival processes for heavy-tailed claim size distributions.

Furthermore, as discussed in Chapter XIII.9 of [1], asymptotic results related to multidimensional ruin problem are to be found in other research areas. For example, $[11,12]$ investigate the asymptotic properties of the hitting probability of a rare set in a multidimensional random walk with light-tailed increments that involves an extension to the ruin problem studied in [13]. A related work in a multidimensional Lévy process is given by [16]. Also, [21] provides the asymptotic behavior of multivariate regularly varying random walks. We refer to [6] for the efficient computation, and to [7] for the asymptotic description of the conditional measure. Also, [20] deals with the heavy-tailed insurance portfolios of several lines of business with possible benefits from diversification effects between businesses. 
In this paper, we seek to derive asymptotic results about conditional expectations given $\tau_{b A}<\infty$ of two risk quantities for both finite and infinite intervals. The first one is the expected accumulated number of claims leading the random walk $S_{n}$ to a rare set that could be different from the ruin set $b A$. The other quantity is the expectation of the total amount of shortfall of $S_{n}$ to a rare set. Note that when quantities of our interest are defined in the discrete time case, the number of claim arrivals asymptotically plays the same role as time in the continuous case as the events $\left\{N_{t} \geq n\right\}$ and $\left\{V_{1}+\cdots+V_{n} \leq t\right\}$ coincide. In view of this discussion, for the continuous-time claim surplus process (2), we retrieve the asymptotic results for the expectations of the total occupation time of $W_{t}$ staying in the rare set and the time-integrated amount of shortfalls to the rare set by utilizing the results in the discrete time case. The precise definitions of these quantities will be provided in the subsequent section. In the literature, similar quantities are considered in the one-dimensional risk model. For example $[23,5]$ study the expected time-integrated negative part of the process under the conditional measure given ruin in continuous time one-dimensional random walks; see also [18] considering the risk reserve process after the surplus is negative until bankruptcy occurs. For discrete-time compound Poisson processes, moments of the time to recovery up to non-ruin level zero was exploited in [17].

The contribution of this paper is two-fold. First, we generalize the one-dimensional results to the multivariate regularly varying random walks. The current setup provides a natural mechanism for incorporating the extremal dependence among the claims under the heavy-tailed setting. Second, the risk quantities are of more general forms and thus it requires different techniques than those in the literature to analyze them. Concerning the rare set that is used to define the risk quantities, it is assumed to be possibly different from the ruin set $b A$. This setting provides more flexibility of implementing the risk quantities under different frameworks of regulatory jurisdiction or internal rules. For example, some changes on the definition of the rare set may be necessary corresponding to the imposition of strong regulatory constraints. This is discussed further in Section 2.1. Under such a setting, the usual exercise of Fubini's theorem is not applicable. Hence we apply a total variation approximation result for the conditional measure $P\left(\cdot \mid \tau_{b A}<\infty\right)$ (c.f. [7]) together with a uniform integrability analysis. For the latter analysis, we employ the technique of Lyapunov inequality to control the tail of the risk quantities under the conditional measure and to ensure the uniform integrability.

The paper is structured as follows. In Section 2.1, we provide the problem setting and in Section 2.2 we give the asymptotic results of risk quantities aforementioned in both discrete time and continuous time cases. The main results given in Theorem 1 and Theorem 2 are proved in Section 3 and Section 4 respectively. 


\section{Main results}

\subsection{Problem setting}

We consider the processes $U_{t}, W_{t}, R_{n}$, and $S_{n}$ defined as in Section 1. Most of the analysis concerns the discrete time process $S_{n}$. To facilitate the discussion, we introduce additional notation $s=0$ that is the starting value of $S_{n}$

$$
S_{n}=s+\sum_{i=1}^{n} X_{i},
$$

where $X_{1}, \ldots, X_{n}$ are i.i.d. increments. Let $X=Y-V c$ be the generic random vector identically distributed with $X_{i}$ and it follows a multivariate regularly varying distribution denoted as $X \in$ $\operatorname{RV}(\alpha, \mu)$. That is, there exists a sequence $\left\{a_{n}: n \geq 1\right\}, 0<a_{n} \uparrow \infty$, and a non-null Radon measure $\mu$ on the compactified and punctured space $\overline{\mathbb{R}}^{d} \backslash\{0\}$ with $\mu\left(\overline{\mathbb{R}}^{d} \backslash \mathbb{R}^{d}\right)=0$ such that, as $n \rightarrow \infty$

$$
n P\left(a_{n}^{-1} X \in \cdot\right) \stackrel{v}{\rightarrow} \mu(\cdot)
$$

where " $\stackrel{v}{\rightarrow}$ " refers to vague convergence. It can be shown that as $b \rightarrow \infty$,

$$
\frac{P(X \in b \cdot)}{P(|X|>b)} \stackrel{v}{\rightarrow} \lambda \mu(\cdot)
$$

for some $c>0$ ([21], Remark 1.1). To simplify notation, $a_{n}$ is chosen such that $n P\left(|X|>a_{n}\right) \rightarrow 1$ and with this choice of $a_{n}$ we have $\lambda=1$. The random vector $X$ has a very small probability of jumping into a set $B$ if $\mu(B)=0$. If $B$ includes an appropriate neighborhood containing the origin, then we can obtain that $\mu(B)=\infty$. Under such a setting, $|X|$ is a regularly varying random variable satisfying $P(|X|>b)=b^{-\alpha} L(b)$ for some $\alpha>0$, where $L(x)$ is a slowly varying function, that is, $L(t b) / L(b) \rightarrow 1$ as $b \rightarrow \infty$ for each $t>0$. Then, we say that $\mu(\cdot)$ has a (regularly varying) index $\alpha$. See [28] for further descriptions of multivariate regular variation. We remark that if $Y \in \operatorname{RV}(\alpha, \mu)$ and $E\left(V^{\alpha+\varepsilon}\right)<\infty$ for some $\varepsilon>0$ then $X \in \operatorname{RV}(\alpha, \mu)$. Let

$$
\eta \triangleq E X
$$

be the mean vector. Throughout this paper we shall use the notation $P_{s}(\cdot)$ for the probability measure on the path space of the process $S=\left(S_{n}: n \geq 0\right)$ given that $S_{0}=s$.

To describe the ruin set, consider $v_{1}, \ldots, v_{m} \in \mathbb{R}^{d}$ and $a_{1}, \ldots, a_{m} \in \mathbb{R}^{+}$. Then define the set $A$ as

$$
A=\cup_{j=1}^{m}\left\{y: y^{\top} v_{j}>a_{j}\right\}=\left\{y: \max _{j=1}^{m}\left(y^{\top} v_{j}-a_{j}\right)>0\right\}
$$

which is the union of $m$ half spaces whose boundaries are hyperplanes. We further introduce

$$
b A=\left\{y: \max _{j=1}^{m}\left(y^{\top} v_{j}-a_{j} b\right)>0\right\}
$$


and the accumulated number of claims to enter this set for the first time is denoted as $\tau_{b A}=$ $\inf \left\{n \geq 0: S_{n} \in b A\right\}$ and its continuous analogue is $\xi_{b A}=\inf \left\{t \geq 0: W_{t} \in b A\right\}$.

In the context of risk theory, the set $A$ can be interpreted as the ruin set for an insurance company holding multiple lines that may be subject to common shocks. We now illustrate two situations for $d=2$.

Situation 1. Consider a company with two business or product lines. A prefixed portion of the reserve of each line can be used to cover the claim surplus of the other line in case one is insolvent (e.g. in a situation that the reserve falls below certain level such as zero). Hence, ruin event for such a company is declared when support from the solvent line is not sufficient to cover the deficit of the insolvent line. Let $y_{i}$ for $i=1,2$ refer as the claim surplus level of the $i$-th business or product line. Their initial reserves are denoted by $R_{0,1}$ and $R_{0,2}$. For the $i$-th business line, let $p_{i} \in[0,1]$ be the proportion of reserve that can be used to cover the severity of the other line. The resulting claim surpluses are monitored at each claim instance whether they are in the ruin set such as

$$
\left\{\left(y_{1}, y_{2}\right): R_{0,1}-y_{1}+p_{2}\left(R_{0,2}-y_{2}\right)<0\right\} \cup\left\{\left(y_{1}, y_{2}\right): p_{1}\left(R_{0,1}-y_{1}\right)+R_{0,2}-y_{2}<0\right\}
$$

This ruin set is in the form of $A$ in (10). Certainly both business lines become insolvent if $y_{1}>R_{0,1}$ and $y_{2}>R_{0,2}$.

Situation 2. Let us assume that $m$ subsidiaries share two products partially. Each subsidiary has an initial reserve $R_{0, j}$ for $j=1, \ldots, m$. Let $y_{i}$ be the claim surplus of the $i$-th product line for $i=1,2$ that is proportionally retained by the $j$-th subsidiary as $p_{i j} y_{i}$ where $p_{i j} \in[0,1]$ and $\sum_{j=1}^{m} p_{i j}=1$. In this case, ruin occurs if one of the $m$ subsidiaries is insolvent, that is

$$
\left\{\left(y_{1}, y_{2}\right): p_{1 j} y_{1}+p_{2 j} y_{2}>R_{0, j}, \text { for some } j\right\}
$$

which is also in the form of $A$ in (10). Further discussions of the modeling of the ruin set $A$ and related topics may be found in [20].

Throughout this paper we consider the conditional expectations given ruin. The following risk quantities are studied. The first one is the accumulated number of claims leading the random walk $S_{n}$ to a set $b C$ from the ruin until $T(b)$ claims as prescribed, that is,

$$
\gamma(b)=\sum_{k=\tau_{b A}}^{T(b)} I\left(S_{k} \in b C\right) .
$$

We write $T(b)$ to emphasize that it possibly depends on $b$. It is also possible that $T(b)=\infty$. We are interested in studying asymptotic approximations for its conditional expectation

$$
\Lambda(b)=E\left[\gamma(b) \mid \tau_{b A}<T(b)\right] .
$$


The second risk quantity is the amount of shortfall of the random walk $S_{n}$ in $b C$ during the interval $\left[\tau_{b A}, T(b)\right]$,

$$
\beta(b)=\sum_{k=\tau_{b A}}^{T(b)} \inf \left\{\|x\|_{1}: S_{k}+x \notin b C\right\},
$$

where $\|\cdot\|_{1}$ denotes the $L_{1}$-distance. Its conditional expectation given ruin is denoted by

$$
\Upsilon(b)=E\left[\beta(b) \mid \tau_{b A}<T(b)\right] .
$$

The expectation $\Upsilon(b)$ can be viewed as the expected amount of reserve one needs to add to the current business in order to be recovered from the financial distress situation defined by set $b C$. The continuous analogues of (11) and (13) are respectively given by

$$
\zeta(b)=\int_{\xi_{b A}}^{v T(b)} I\left(W_{t} \in b C\right) d t, \quad \theta(b)=\int_{\xi_{b A}}^{v T(b)} \inf \left\{\|x\|_{1}: W_{t}+x \notin b C\right\} d t
$$

where $v=E(V)$. They are interpreted as the total occupation time of $W_{t}$ staying in a rare set, and time-integrated amount of shortfalls to a rare set during the interval $\left[\xi_{b A}, v T(b)\right]$. The analysis of these quantities are motivated by $[23,5]$ who have studied the expected value of the total time below 0 , and the expected value of the time-integrated negative part of the risk process for a fixed time $T$ under one-dimensional risk model in continuous time. They are defined respectively as

$\int_{0}^{T} I\left(U_{t}<0\right) d t$ and $\int_{0}^{T}\left|U_{t}\right| I\left(U_{t}<0\right) d t$ which are of interest in connection with the risk measures with a finite time horizon in a insurance regulation.

We would like to provide some comments on the rare set $b C$. If $C=A$, then (12) and (14) are of interest as generalizations of $[23,5]$ in discrete model, and by means of Fubini's theorem the asymptotic results for (12) and (14) can be derived. If $C \neq A$, then the situation is more complicated and alternative techniques are necessary. Due to managerial or regulatory purpose, (12) and (14) may be analyzed with a judicious choice of the rare set $b C$ which is possibly different from the ruin set $b A$. Very often, we make $A$ be a strict subset of $C$ to make sure that the business is prudently managed after its operation faced financial difficulty (i.e. the surplus level hit certain ruin set $A$ ). For instance, in Situation 1, we may choose $C=\left\{\left(y_{1}, y_{2}\right): y_{i}>R_{0, i}\right.$ for $\left.i=1,2\right\}$, that is, the reserve of each business line recovers to the positive level without the aid from the other one. Furthermore, we may consider more strict regulations such as $C=\left\{\left(y_{1}, y_{2}\right): y_{i}>(1-\delta) R_{0, i}\right.$ for $\left.i=1,2\right\}$, that is, $\delta$ fraction of the initial reserve must hold as reserve which is set by rule. Our asymptotic results for (12) and (14) as well as expectations of (15) derived in the following sections are applicable to these more generalized settings.

\subsection{The main theorems}

To obtain the asymptotic approximations, we need to impose the following technical assumptions. 
Assumption 1 The increment $X$ follows a continuous multivariate regularly varying distribution with tail index $\alpha$ and the drift $\eta=-\mathbf{1}$, where $\mathbf{1}=(1, \ldots, 1)^{\top} \in \mathbb{R}^{d}$.

Assumption 2 For each $j, \eta^{\top} v_{j}=-1$ and $\mu\left(\left\{y: y^{\top} v_{j}\right\}\right)>0$ for all $j$.

Assumption 3 The time $T(b)$ admits the asymptotic behavior that

$$
\lim _{b \rightarrow \infty} \frac{T(b)}{b}=T^{*} \in(0, \infty]
$$

Assumption 4 The set $b C$ grows linearly with $b$, where $C$ is a union of half spaces

$$
C=\left\{y: \max _{j=1}^{m^{\prime}}\left(y^{\top} v_{j}^{\prime}-a_{j}^{\prime}\right)>0\right\} \quad \text { such that } a_{j}^{\prime}>0, v_{j}^{\prime} \in \mathbb{R}^{d} \text {, and } \eta^{\top} v_{j}^{\prime}<0,
$$

and $\mu\left(\left\{y: y^{\top} v_{j}^{\prime}\right\}\right)>0$ for all $j$.

In Assumptions 1 and 2, we constrain that $\eta=-\mathbf{1}$ and $\eta^{\top} v_{j}=-1$. These two constraints are imposed simply for technical and notational convenience. Indeed, for any nonzero drift $\eta^{\prime}$, we can always find a non-degenerate matrix $M$ such that $M \eta^{\prime}=\mathbf{- 1}$. This implies that an affine transformation of the space does not alter the problem and thus Assumption 1 does not impose any essential restriction on the drift function. Furthermore, suppose that $\eta^{\top} \tilde{v}_{j}=c_{j}<0$ and the corresponding boundary of $A$ is $y^{\top} \tilde{v}_{j}>\tilde{a}_{j}$. Then we can always rescale the vector $v_{j}=\tilde{v}_{j} /\left|c_{j}\right|$ and $a_{j}=\tilde{a}_{j} /\left|c_{j}\right|$ so that $\eta^{\top} v_{j}=-1$. Nonetheless, it is important that $\eta^{\top} v_{j}<0$, otherwise, the random walk hits the ruin set $b A$ with probability one no matter how large $b$ is. In addition, $\mu\left(\left\{y: y^{\top} v_{j}\right\}\right)>0$ is a condition to rule out the degenerated cases.

The statements of the theorems need the following construction. For each $z>0$, let $Y(z)$ be a random vector with distribution

$$
P(Y(z) \in B)=\frac{\mu\left(B \cap\left\{y: \max _{j=1}^{m}\left[y^{\top} v_{j}-a_{j}\right] \geq z\right\}\right)}{\mu\left(\left\{y: \max _{j=1}^{m}\left[y^{\top} v_{j}-a_{j}\right] \geq z\right\}\right)},
$$

where $\mu$ is the limiting measure defined as in (7). The random vector $Y(z)$ asymptotically follows the conditional distribution $P\left(b^{-1} X \in \cdot \mid \max _{j=1}^{m}\left[X^{\top} v_{j}-a_{j} b\right] \geq z b\right)$. According to the "one-bigjump" principle, the random walk reaches the target set $b A$ due to one big jump, while the rest of the increment largely follow their original behavior. Suppose that $S_{n}$ reaches set $b A$ at step $\tau_{b A}=z b+1$. Then, on the scale described by the Law of Large Numbers, random walk $S_{n}$ follows approximately the fluid path before hitting the set $b A$, that is,

$$
S_{n} \approx n \eta \quad \text { for } \quad n<\tau_{b A}
$$

Thus, $X_{\tau_{b A}}$ follows approximately the conditional distribution

$$
P\left(X \in \cdot \mid \max _{j=1}^{m}\left[X^{\top} v_{j}-a_{j} b\right] \geq \tau_{b A}\right) .
$$


Therefore, if $z=\tau_{b A} / b$ then $Y(z)$ approximates the increment at the instance of ruin (scaled down by a factor $b$ ). In addition, asymptotic distribution of $\tau_{b A}$ is given as follows. By the result of the regularly variation, we can define for any $a_{1}, \ldots, a_{m}>0$

$$
\lim _{b \rightarrow \infty} \frac{P\left(\max _{j=1}^{m}\left[X^{\top} v_{j}-a_{j} b\right]>0\right)}{P(|X|>b)}=\kappa\left(a_{1}, \ldots, a_{m}\right) .
$$

Recall the definition of the limit measure $\mu$, it is straightforward to verify that

$$
\kappa\left(a_{1}, \ldots, a_{m}\right) \triangleq \mu\left(\left\{y: \max _{j=1}^{m}\left(y^{\top} v_{j}-a_{j}\right)>0\right\}\right)
$$

To simplify the notation we write

$$
\kappa_{\mathbf{a}}(t)=\kappa\left(a_{1}+t, \ldots, a_{m}+t\right)
$$

where $\mathbf{a}=\left(a_{1}, \ldots, a_{m}\right)$. Let $Z$ be a positive random variable following the distribution

$$
P(Z>t)=\exp \left\{-\int_{0}^{t} \frac{\kappa_{\mathbf{a}}(s)}{\int_{s}^{\infty} \kappa_{\mathbf{a}}(u) d u} d s\right\}, \quad t \geq 0
$$

where $\kappa_{\mathbf{a}}(\cdot)$ is as defined in (18). The random variable $Z$ approximates $\tau_{b A}$ in the sense that

$$
\frac{\tau_{b A}}{b} \Rightarrow Z
$$

as $b \rightarrow \infty$ where " $\Rightarrow$ " denotes weak convergence. Based on these constructions, we present two theorems containing the asymptotic results for the risk quantities defined in (12) and (14). In turn, we obtain asymptotic results for the expectations of (15) as well. Our main results are presented as follows.

Theorem 1 Under the Assumptions 1-4, for $z \leq T^{*}$, we define function

$$
s_{\gamma}(x, z)=\int_{0}^{T^{*}-z} I(x+t \eta \in C) d t .
$$

If the tail index $\alpha>2$, then (12) is asymptotically approximated as

$$
\lim _{b \rightarrow \infty} \frac{\Lambda(b)}{b}=E\left[s_{\gamma}(Y(Z)+\eta Z, Z) \mid Z \leq T^{*}\right]
$$

where the the conditional distribution of $Y(Z)$ given $Z=z$ is provided in (16), and the distribution of $Z$ is given in (19).

Continuing the previous discussion, under the conditional measure $P\left(\cdot \mid \tau_{b A}<\infty\right)$, the first passage time and the overshoot follow the asymptotic distribution that

$$
\left(\frac{\tau_{b A}}{b}, \frac{X_{\tau_{b A}}}{b}\right) \Rightarrow(Z, Y(Z))
$$


Furthermore, for each $x \notin C$, the ray $\{x+\eta t: t \geq 0\}$, which is the path of the random walk on the fluid scale described by the Law of Large Numbers, does not intersect the rare set $C$. Thus, $s_{\gamma}(x, z)=0$ for all $x \notin C$. We scale the space and time by $b^{-1}$. For $x \in C$ corresponding to the first landing position of the random walk in the rare set at time $z$, the function $s_{\gamma}(x, z)$ measures the amount of time during which the ray $\{x+\eta t: t \geq 0\}$ stays in the rare set prior to $T^{*}$. Therefore, the random variable $s_{\gamma}(Y(Z)+\eta Z, Z)$ is the asymptotic total number of claim arrivals while the random walk is in the set $b C$.

Theorem 2 Under the Assumptions 1-4, we define function

$$
s_{\beta}(x, z)=\int_{0}^{T^{*}-z} \inf \left\{\|y\|_{1}: x+t \eta+y \notin C\right\} d t .
$$

If the tail index $\alpha>3$, then (14) is asymptotically obtained as

$$
\lim _{b \rightarrow \infty} \frac{\Upsilon(b)}{b^{2}}=E\left[s_{\beta}(Y(Z)+\eta Z, Z) \mid Z \leq T^{*}\right] .
$$

Note that the function $s_{\beta}$ has a similar interpretation corresponding to $\beta(b)$ as that of $s_{\gamma}$ corresponding to $\gamma(b)$.

Remark 1 For the case $T^{*}=\infty$, the asymptotic results for the conditional expectations of $\gamma(b)$ and $\beta(b)$ for $\tau_{b A}<\infty$ are also obtained as special cases of the above theorems. We basically replace $T^{*}$ in the definitions of $s_{\gamma}$ and $s_{\beta}$ by infinity, that is, $s_{\gamma}=\int_{0}^{\infty} I(x+t \eta \in C) d t$ and $s_{\beta}=\int_{0}^{\infty} \inf \left\{\|y\|_{1}: x+t \eta+y \notin C\right\} d t$. Then one finds

$$
\lim _{b \rightarrow \infty} E\left[\gamma(b) / b \mid \tau_{b A}<\infty\right]=E\left[s_{\gamma}(Y(Z)+\eta Z, Z)\right],
$$

and

$$
\lim _{b \rightarrow \infty} E\left[\beta(b) / b^{2} \mid \tau_{b A}<\infty\right]=E\left[s_{\beta}(Y(Z)+\eta Z, Z)\right] .
$$

As a corollary of the above two theorems, the corresponding results for the continuous-time risk quantities given in (15) are presented in the following.

Corollary 1 Let $v=E(V)$. Under the Assumptions 1-4, if $\alpha>2$, then

$$
\lim _{b \rightarrow \infty} \frac{E\left[\zeta(b) \mid \xi_{b A} / v<T(b)\right]}{v b}=E\left[s_{\gamma}(Y(Z)+\eta Z, Z)\right]
$$

and if $\alpha>3$, then

$$
\lim _{b \rightarrow \infty} \frac{E\left[\theta(b) \mid \xi_{b A} / v<T(b)\right]}{v b^{2}}=E\left[s_{\beta}(Y(Z)+\eta Z, Z)\right]
$$


The proof of the above corollary is completely analogous to those of Theorems 1 and 2 . Therefore, we omit the details and only provide an intuitive argument. According to identity (6), $\tau_{b A}$ and $\xi_{b A}$ are respectively the total number of claims and the time at which $S_{n}$ and $W_{t}$ enter set $b A$ for the first time. By the law of large numbers, it yields that $\xi_{b A} / \tau_{b A} \rightarrow v$ as $b \rightarrow \infty$. Then, the conditional measures admit the approximation $P\left(\cdot \mid \tau_{b A}<T(b)\right) \approx P\left(\cdot \mid \xi_{b A} / v<T(b)\right)$. Furthermore, note that $\gamma(b)$ is the total number of claims from $\tau_{b A}$ to $T(b)$, and $\zeta(b)$ is the total amount of time from $\xi_{b A}$ to $v T(b)$. On average, one claim arrives in very $v$ amount of time. Therefore, we establish the connection $v \gamma(b) \approx \zeta(b)$ and similarly $v \beta(b) \approx \theta(b)$. To obtain the corollary based on the theorems given previously, we basically replace $\tau_{b A}$ by $v \xi_{b A}$ and scale up the approximations by the factor $v$. All the above heuristic arguments can be made rigorous.

\section{Proof of Theorem 1}

To begin, define a localization set for a large $\lambda$ (which is specified later)

$$
L_{\lambda}(b)=\cap_{j=1}^{m^{\prime}}\left\{y: y^{\top} v_{j}^{\prime}<\lambda b a_{j}^{\prime}\right\}
$$

where $v_{j}^{\prime}$ 's are the vectors defining set $C$. Using $L_{\lambda}(b)$, we decompose the conditional expectation of (11) into a sum of two terms, namely

$$
E\left[\gamma(b) \mid \tau_{b A}<T(b)\right]=E\left[\gamma(b) ; S_{\tau_{b A}} \in L_{\lambda}(b) \mid \tau_{b A}<T(b)\right]+E\left[\gamma(b) ; S_{\tau_{b A}} \in L_{\lambda}^{c}(b) \mid \tau_{b A}<T(b)\right]
$$

The first term on the right-hand side of (22) equals

$$
E\left[\gamma(b) ; S_{\tau_{b A}} \in L_{\lambda}(b) \mid \tau_{b A}<T(b)\right]=E\left[E_{S_{\tau_{b A}}}[\gamma(b)] ; S_{\tau_{b A}} \in L_{\lambda}(b) \mid \tau_{b A}<T(b)\right]
$$

where $E_{s}(\cdot)$ is the conditional expectation given that $S_{0}=s$. The following lemma provides a bound of $E_{s}[\gamma(b)]$ for $s \in L_{\lambda}(b)$.

Lemma 1 For each $\lambda$, there exists a $\kappa_{0}$ such that

$$
E_{s}[\gamma(b)] \leq \kappa_{0} \lambda b
$$

for all $s \in L_{\lambda}(b)$.

Proof of Lemma 1. For each $s \in L_{\lambda}(b)$, using Fubini's theorem results in

$$
E_{s}[\gamma(b)] \leq E_{s}\left[\sum_{k=1}^{\infty} I\left(S_{k} \in b C\right)\right] \leq \sum_{k=1}^{\infty} P_{s}\left(S_{k} \in b C\right)
$$

where

$$
\left\{S_{k} \in b C\right\}=\cup_{j=1}^{m^{\prime}}\left\{s^{\top} v_{j}^{\prime}+\sum_{i=1}^{k} X_{i}^{\top} v_{j}^{\prime}>b a_{j}^{\prime}\right\} .
$$


In addition, for each $j$, note that $X_{i}^{\top} v_{j}^{\prime}$ for $i=1,2, \ldots$ are i.i.d. univariate random variables with expectation $E X_{i}^{\top} v_{j}^{\prime}<0$, and hence we can obtain two-sided bounds for $P_{s}\left(S_{k} \in b C\right)$ as

$$
\begin{aligned}
\max _{j=1, \ldots, m^{\prime}} & P_{s}\left(s^{\top} v_{j}^{\prime}+\sum_{i=1}^{k} X_{i}^{\top} v_{j}^{\prime}>b a_{j}^{\prime}\right) \leq P_{s}\left(S_{k} \in b C\right) \\
& \leq \sum_{j=1}^{m^{\prime}} P_{s}\left(s^{\top} v_{j}^{\prime}+\sum_{i=1}^{k} X_{i}^{\top} v_{j}^{\prime}>b a_{j}^{\prime}\right) \leq \max _{j=1, \ldots, m^{\prime}} m^{\prime} \times P_{s}\left(s^{\top} v_{j}^{\prime}+\sum_{i=1}^{k} X_{i}^{\top} v_{j}^{\prime}>b a_{j}^{\prime}\right) .
\end{aligned}
$$

Let $j$ be the maximizer of the right-hand side of the above display. Since $X_{i}$ 's are multivariate regularly varying random vectors and $\mu(C)>0$, according to the classic result of [4], $X_{i}^{\top} v_{j}^{\prime}$ 's are all regularly varying random vectors. Define

$$
-\delta_{0}=\min _{j} E\left(X_{i}^{\top} v_{j}^{\prime}\right)<0
$$

which leads to the representation

$$
P_{s}\left(s^{\top} v_{j}^{\prime}+\sum_{i=1}^{k} X_{i}^{\top} v_{j}^{\prime}>b a_{j}^{\prime}\right)=P_{s}\left(\sum_{i=1}^{k}\left(X_{i}^{\top} v_{j}^{\prime}+\delta_{0}\right)>b a_{j}^{\prime}+k \delta_{0}-s^{\top} v_{j}^{\prime}\right),
$$

and recall that $s^{\top} v_{j}^{\prime}<\lambda b a_{j}^{\prime}$ for each $s \in L_{\lambda}(b)$. Then, for $k>2 \delta_{0}^{-1} \lambda b \max _{j} a_{j}^{\prime}$, we have

$$
b a_{j}^{\prime}+k \delta_{0}-s^{\top} v_{j}^{\prime}>k \delta_{0} / 3
$$

By the standard large deviations results of regularly varying random variables (e.g. [24, 25, 26]), it follows that

$P_{s}\left(s^{\top} v_{j}^{\prime}+\sum_{i=1}^{k} X_{i}^{\top} v_{j}^{\prime}>b a_{j}^{\prime}\right) \sim k \times P\left(X^{\top} v_{j}^{\prime}+\delta_{0}>b a_{j}^{\prime}+k \delta_{0}-s^{\top} v_{j}^{\prime}\right) \leq k \times P\left(X^{\top} v_{j}^{\prime}+\delta_{0}>k \delta_{0} / 3\right)$.

Then, there exists a $\kappa_{1}$ such that

$$
\begin{aligned}
P_{s}\left(S_{k} \in b C\right) & \leq \sum_{j=1}^{m^{\prime}} k P\left(X^{\top} v_{j}^{\prime}>k \delta_{0} / 3\right) \\
& \leq \kappa_{1} L(k) k^{-\alpha+1}
\end{aligned}
$$

for all $k>2 \delta_{0}^{-1} \lambda b \max _{j} a_{j}^{\prime}$. Therefore,

$$
\begin{aligned}
\sum_{k=1}^{\infty} P_{s}\left(S_{k} \in b C\right) & \leq 2 \delta_{0}^{-1} \lambda b \max _{j} a_{j}^{\prime}+\sum_{k=2 \delta_{0}^{-1} \lambda b \max _{j} a_{j}^{\prime}+1}^{\infty} \kappa_{1} L(k) k^{-\alpha+1} \\
& \leq \kappa_{0} \lambda b
\end{aligned}
$$

and the result follows.

The following proposition, given by Theorem 2 in [7], provides a useful weak convergence result for our analysis. 
Proposition 1 Under the conditions of Theorem 1, we have that the following weak convergence result conditional on $\tau_{b A}<\infty$,

$$
\left(\frac{\tau_{b A}}{b}, \frac{X_{\tau_{b A}}}{b}, \frac{S_{\tau_{b A}}}{b}\right) \Rightarrow(Z, Y(Z), Y(Z)+\eta Z) \quad \text { as } b \rightarrow \infty
$$

in $\mathbb{R} \times \mathbb{R}^{d} \times \mathbb{R}^{d}$. The distributions for $Y$ and $Z$ are given in (16) and (19) respectively.

Note that the conditional expectation $E_{s}[\gamma(b) / b]$ is bounded for all $s \in L_{\lambda}(b)$ from Lemma 1 , and the weak convergence result suggests that there exists $\varepsilon_{0}>0$ such that $P\left(\tau_{b A}<T(b) \mid \tau_{b A}<\infty\right)>\varepsilon_{0}$. Thus, an approximation of $E\left[\gamma(b) ; S_{\tau_{b A}} \in L_{\lambda}(b) \mid \tau_{b A}<T(b)\right]$ can be obtained

$$
\begin{aligned}
E\left[\gamma(b) / b ; S_{\tau_{b A}}\right. & \left.\in L_{\lambda}(b) \mid \tau_{b A}<T(b)\right] \\
& \rightarrow E\left[s_{\gamma}(Y(Z)+\eta Z, Z) ; b Y(Z)+b \eta Z \in L_{\lambda}(b) \mid Z<T^{*}\right]
\end{aligned}
$$

as $b \rightarrow \infty$. The above convergence is founded by replacing the random walk $S_{\tau_{b A}+k}$ by its fluid limit $S_{\tau_{b A}+k} \approx S_{\tau_{b A}}+k \eta$ and using the approximation result $S_{\tau_{b A}} / b \approx Y(Z)+\eta Z$. Then by monotone convergence theorem, the right-hand side of the above limit tends to

$$
E\left[s_{\gamma}(Y(Z)+\eta Z, Z) ; b Y(Z)+b \eta Z \in L_{\lambda}(b) \mid Z<T^{*}\right] \rightarrow E\left[s_{\gamma}(Y(Z)+\eta Z, Z) \mid Z<T^{*}\right]
$$

as $\lambda \rightarrow \infty$.

Now it remains to show that the second term of (22) is ignorable for large $\lambda$. To do this, let us first denote

$$
\tau_{L^{c}}=\inf \left\{k \geq 0: S_{k} \in L_{\lambda}^{c}(b)\right\}
$$

which represents the number of claims leading the random walk $S_{n}$ to the complement set of $L_{\lambda}(b)$ for first time. Then, one finds

$$
\begin{aligned}
E\left[\gamma(b) ; S_{\tau_{b A}} \in L_{\lambda}^{c}(b) ; \tau_{b A}<T(b)\right] & \leq E\left[\gamma(b) ; \tau_{L^{c}} \leq \tau_{b A}<T(b)\right] \\
& \leq E\left[\sum_{k=\tau_{L^{c}}}^{T(b)} I\left(S_{k} \in b C\right) ; \tau_{L^{c}}<T(b)\right] .
\end{aligned}
$$

The following lemma facilitates to establish a bound for (24) and its proof can be found in [6].

Lemma 2 Suppose that there exists a nonnegative function $g_{b}(s)$ such that for $s \notin B$,

$$
E\left[g_{b}(s+X)\right] \leq g_{b}(s)
$$

and for $s \in B, g_{b}(s) \geq \varepsilon l(s)$. Then, for $s \notin B$,

$$
E\left[l\left(S_{\tau_{B}}\right) \mid S_{0}=s\right] \leq \varepsilon^{-1} g_{b}(s),
$$

where $\tau_{B}=\inf \left\{k: S_{k} \in B\right\}$. 


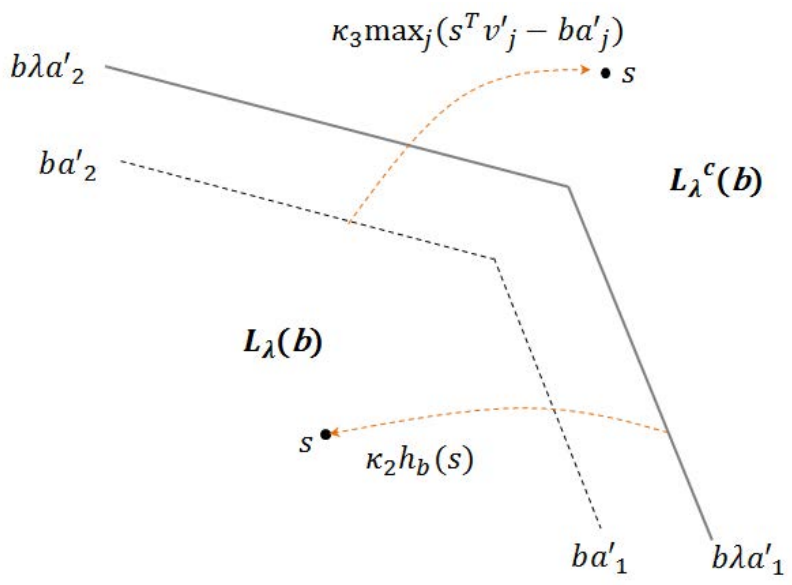

Figure 1: Illustration of a Lyapunov function for two-dimensional random walk

We refer the inequality in (25) as the Lyapunov inequality and $g_{b}(s)$ as a Lyapunov function that provide a means to develop an upper bound for (24). The rest of the proof is dedicated to the establishment of the Lyapunov inequality by constructing an appropriate Lyapunov function with $B \approx L_{\lambda}^{c}(b)$ and $l(s)=E\left[\sum_{k=\tau_{L^{c}}}^{T(b)} I\left(S_{k} \in b C\right) \mid S_{\tau_{L^{c}}}=s\right]$.

To construct a Lyapunov function, let us define the integrated tail

$$
G_{j}(x)=\int_{x}^{\infty} P\left(X^{\top} v_{j}^{\prime}>t\right) d t
$$

and

$$
h_{b}(s)=\sum_{j=1}^{m^{\prime}}\left(\kappa b \lambda+b \lambda a_{j}^{\prime}-s^{\top} v_{j}^{\prime}\right) G_{j}\left(b \lambda a_{j}^{\prime}-s^{\top} v_{j}^{\prime}\right)
$$

for some $\kappa>0$ (to be determined later). Lastly, we construct a candidate of Lyapunov function as

$$
g_{b}(s)=\left\{\begin{array}{ll}
\min \left(\kappa_{2} h_{b}(s), m^{\prime} \kappa b \lambda\right) & \text { if } s \in L_{\lambda}(b) \\
\max \left(\kappa_{3} \max _{j}\left(s^{\top} v_{j}^{\prime}-b a_{j}^{\prime}\right), m^{\prime} \kappa b \lambda\right) & \text { if } s \in L_{\lambda}^{c}(b)
\end{array} .\right.
$$

For the two-dimensional case, in Figure 1 the above Lyapunov function and the level sets are illustrated and $g_{b}(s)$ takes the value of $m^{\prime} \kappa b \lambda$ along the solid line. In addition, the following lemma presents the boundary condition of $g_{b}(s)$.

Lemma 3 By choosing $\kappa$ and $\kappa_{3}$ large, we have that

$$
g_{b}(s) \geq E_{s}\left[\sum_{k=\tau_{L^{c}}}^{T(b)} I\left(S_{k} \in b C\right) ; \tau_{L^{c}}<T(b)\right]
$$

for $s$ such that $g_{b}(s) \geq m^{\prime} \kappa b \lambda$. 
Proof of Lemma 3. The proof is completely analogous to that of Lemma 1 with an application of the Fubini's Theorem and therefore is omitted.

In what follows, we will show that $g_{b}(s)$ satisfies the Lyapunov inequality so that $g_{b}(0)$ is an upper bound of (24). That is, we need to verify

$$
g_{b}(s) \geq E\left[g_{b}(s+X)\right] \quad \text { for } g_{b}(s)<m^{\prime} \kappa b \lambda .
$$

The right-hand side of (27) may be re-expressed as

$$
\begin{aligned}
& E\left[g_{b}(s+X)\right] \\
& \quad=E\left[g_{b}(s+X) ; X \notin C_{a}(s)\right]+E\left[g_{b}(s+X) ; X \in C_{a}(s)\right] \\
& \quad=J_{1}+J_{2},
\end{aligned}
$$

where

$$
C_{a}(s)=\cup_{j=1}^{m^{\prime}}\left\{y: y^{\top} v_{j}^{\prime}>a\left(b \lambda a_{j}^{\prime}-s^{\top} v_{j}^{\prime}\right)\right\} \quad \text { for some } a \in(0,1) .
$$

In the following, let us study $J_{1}$ and $J_{2}$ respectively. Consider an $s$ such that $g_{b}(s)<m^{\prime} \kappa b \lambda$, which implies that $s$ reasonably far away from the set $L_{\lambda}^{c}(b)$. In particular, we can choose $\kappa_{2}$ large such that there exists a $b_{0}$ satisfying

$$
b \lambda a_{j}^{\prime}-s^{\top} v_{j}^{\prime}>b_{0}
$$

for all $g_{b}(s)<m^{\prime} \kappa b \lambda$. First, an upper bound of $J_{2}$ can be obtained in a straightforward manner as follows. Note that for some $\kappa_{4}$ and $\zeta>0$ (independent of $\lambda$, and the choices of $\zeta$ depends on the tail index),

$$
\begin{aligned}
J_{2}=E & {\left[g_{b}(s+X) \mid X \in C_{a}(s)\right] P\left[X \in C_{a}(s)\right] } \\
& \leq \kappa_{4}\left[m^{\prime} \kappa b \lambda+\sum_{j=1}^{m^{\prime}} \zeta \kappa_{3}\left(b \lambda a_{j}^{\prime}-s^{\top} v_{j}^{\prime}\right) w_{j}\right] P\left[X \in C_{a}(s)\right],
\end{aligned}
$$

where

$$
w_{j}=\frac{P\left(X^{\top} v_{j}^{\prime} \geq a\left(b \lambda a_{j}^{\prime}-s^{\top} v_{j}^{\prime}\right)\right)}{\sum_{i=1}^{m^{\prime}} P\left(X^{\top} v_{i}^{\prime} \geq a\left(b \lambda a_{i}^{\prime}-s^{\top} v_{i}^{\prime}\right)\right)} .
$$

Regarding $J_{1}$, using Rolle's representation it may be rewritten as

$$
\begin{aligned}
J_{1} & =E\left[g_{b}(s+X) ; X \notin C_{a}(s)\right] \\
& =g_{b}(s)+E\left[X^{\top} \partial g_{b}(s+\theta X) ; X \notin C_{a}(s)\right],
\end{aligned}
$$

where $\partial g$ is the gradient and $\theta \in(0,1)$ may depend on $X$. We now take a closer look at the gradient when $g_{b}(s)<m^{\prime} \kappa b \lambda$, and then we get

$$
\partial g_{b}(s)=\kappa_{2} \sum_{j=1}^{m^{\prime}}\left[-G_{j}\left(b \lambda a_{j}^{\prime}-s^{\top} v_{j}^{\prime}\right)+\left(\kappa \lambda b+b \lambda a_{j}^{\prime}-s^{\top} v_{j}^{\prime}\right) P\left(X^{\top} v_{j}^{\prime}>b \lambda a_{j}^{\prime}-s^{\top} v_{j}^{\prime}\right)\right] v_{j}^{\prime} .
$$


As a consequence of the regularly variation ( $\alpha$ being the regularly varying tail index), it follows that

$$
\lim _{x \rightarrow \infty} \frac{G_{j}(x)}{x P\left(X^{\top} v_{j}^{\prime}>x\right)}=\frac{1}{\alpha-1} .
$$

We choose $\kappa_{2}$ sufficiently large (independent of $\left.b\right)$ such that $\min _{j}\left(b \lambda a_{j}^{\prime}-s^{\top} v_{j}^{\prime}\right)$ is large on the set $g_{b}(s)<m^{\prime} \kappa \lambda b$, so that

$$
\partial g_{b}(s)=\kappa_{2} \sum_{j=1}^{m^{\prime}}\left[\kappa \lambda b P\left(X^{\top} v_{j}^{\prime}>b \lambda a_{j}^{\prime}-s^{\top} v_{j}^{\prime}\right)+\{\alpha-2+o(1)\} G\left(b \lambda a_{j}^{\prime}-s^{\top} v_{j}^{\prime}\right)\right] v_{j}^{\prime},
$$

where the $o(1)$ is with respect to $\min _{j}\left(b \lambda a_{j}^{\prime}-s^{\top} v_{j}^{\prime}\right) \rightarrow \infty$. According to regularly variation, for each $j$ and $x \in C_{a}(s)$, there exists a $\kappa_{5}>0$ such that

$$
P\left(X^{\top} v_{j}^{\prime}>b \lambda a_{j}^{\prime}-(s+x)^{\top} v_{j}^{\prime}\right) \leq \kappa_{5} P\left(X^{\top} v_{j}^{\prime}>b \lambda a_{j}^{\prime}-s^{\top} v_{j}^{\prime}\right) .
$$

By means of the dominated convergence theorem, for a small positive constant $\varepsilon_{0}>0$ and sufficiently large $\min _{j}\left(b \lambda a_{j}^{\prime}-s^{\top} v_{j}^{\prime}\right)$, the second term on the right-hand side of (30) is bounded by

$$
\begin{aligned}
& E\left[X^{\top} \partial g_{b}(s+\theta X) ; X \notin C_{a}(s)\right] \\
& \quad \leq-\delta_{0}\left(\kappa_{2}-\varepsilon_{0}\right) \sum_{j=1}^{m^{\prime}}\left[\kappa b \lambda P\left(X^{\top} v_{j}^{\prime}>b \lambda a_{j}^{\prime}-s^{\top} v_{j}^{\prime}\right)+\{\alpha-2+o(1)\} G_{j}\left(b \lambda a_{j}^{\prime}-s^{\top} v_{j}^{\prime}\right)\right],
\end{aligned}
$$

where $-\delta_{0}=\min _{j} E\left(X^{\top} v_{j}^{\prime}\right)<0$, which can be achieved by choosing $\kappa_{2}$ sufficiently large. Comparing the right-hand side of the above bound and $J_{2}$ in (29), we can always find $\kappa_{2}$ large enough with sufficiently small $\varepsilon_{0}$, so that

$$
\begin{gathered}
\delta_{0}\left(\kappa_{2}-\varepsilon_{0}\right) \sum_{j=1}^{m^{\prime}}\left[\kappa b \lambda P\left(X^{\top} v_{j}^{\prime}>b \lambda a_{j}^{\prime}-s^{\top} v_{j}^{\prime}\right)+\{\alpha-2+o(1)\} G_{j}\left(b \lambda a_{j}^{\prime}-s^{\top} v_{j}^{\prime}\right)\right] \\
>\kappa_{4}\left[m^{\prime} \kappa b \lambda+\sum_{j=1}^{m^{\prime}} \zeta \kappa_{3}\left(b \lambda a_{j}^{\prime}-s^{\top} v_{j}^{\prime}\right) w_{j}\right] P\left[X \in C_{a}(s)\right] .
\end{gathered}
$$

But $P\left[X \in C_{a}(s)\right] \leq \kappa_{6} \sum_{j=1}^{m^{\prime}} P\left(X^{\top} v_{j}^{\prime}>b \lambda a_{j}^{\prime}-s^{\top} v_{j}^{\prime}\right)$, one finds

$$
E\left[X^{\top} \partial g_{b}(s+\theta X) ; X \notin C_{a}(s)\right]+J_{2}<0,
$$

and in turn, from (30)

$$
J_{1}+J_{2} \leq g_{b}(s) .
$$

Thus, the Lyapunov inequality is well established. Then applying Lemma 2 when $B=\left\{s: g_{b}(s) \geq\right.$ $\left.m^{\prime} \kappa b \lambda\right\}$ together with the boundary condition in Lemma 3 results in

$$
E\left[\sum_{k=\tau_{L^{c}}}^{T(b)} I\left(S_{k} \in b C\right) ; \tau_{L^{c}}<T(b)\right] \leq g_{b}(0) \leq O(1) \kappa_{2} L(\lambda b)(\lambda b)^{-\alpha+2} .
$$


According to the asymptotic results in [21], it gives $P\left(\tau_{b A}<\infty\right) \sim \kappa L(b) b^{-\alpha+1}$. Furthermore, in light of Proposition 1, it implies that $P\left(\tau_{b A}<T(b) \mid \tau_{b A}<\infty\right) \geq \varepsilon_{0}$. Hence, one finds

$$
\frac{E\left[\sum_{k=\tau_{L^{c}}}^{T(b)} I\left(S_{k} \in b C\right) ; \tau_{L^{c}}<T(b)\right]}{P\left(\tau_{b A}<T(b)\right)}=O(1) \kappa_{2} \lambda^{-\alpha+2} b .
$$

Note that the above bound becomes $o(b)$ as $\lambda$ tends to be infinity. Combining with the results in (23) concludes the proof.

\section{Proof of Theorem 2}

In a manner which is similar to that in the proof of Theorem 1, with the same set $L_{\lambda}(b)$ defined in the previous section, one has the decomposition

$$
E\left[\beta(b) \mid \tau_{b A}<T(b)\right]=E\left[\beta(b) ; S_{\tau_{b A}} \in L_{\lambda}(b) \mid \tau_{b A}<T(b)\right]+E\left[\beta(b) ; S_{\tau_{b A}} \in L_{\lambda}^{c}(b) \mid \tau_{b A}<T(b)\right] .
$$

The first term on the right-hand side of (32) can be re-expressed as

$$
E\left[\beta(b) ; S_{\tau_{b A}} \in L_{\lambda}(b) \mid \tau_{b A}<T(b)\right]=E\left[E_{S_{\tau_{b A}}}(\beta(b)) ; S_{\tau_{b A}} \in L_{\lambda}(b) \mid \tau_{b A}<T(b)\right] .
$$

The following lemma shows that $E_{s}\left[\beta(b) / b^{2}\right]$ is a bounded function for all $s \in L_{\lambda}(b)$.

Lemma 4 For each $\lambda$, there exists a $\kappa_{0}$ such that

$$
E_{s}[\beta(b)] \leq \kappa_{0} \lambda^{2} b^{2}
$$

for all $s \in L_{\lambda}(b)$.

Proof of Lemma 4. According to the Fubini's theorem, we have that for all $s \in L_{\lambda}(b)$,

$$
\begin{aligned}
E_{s}[\beta(b)] & =E_{s}\left[\sum_{k=1}^{T(b)} \inf \left\{\|x\|_{1}: S_{k}+x \notin b C\right\}\right] \\
& \leq \sum_{k=1}^{\infty} E_{s}\left[\inf \left\{\|x\|_{1}: S_{k}+x \notin b C\right\}\right]
\end{aligned}
$$

Once again, we split the above summation

$$
\begin{aligned}
\sum_{k=1}^{\infty} E_{s}\left[\inf \left\{\|x\|_{1}: S_{k}+x \notin b C\right\}\right] \leq & \sum_{k=1}^{2 \delta_{0}^{-1} b \lambda \max a_{j}^{\prime}} E_{s}\left[\inf \left\{\|x\|_{1}: S_{k}+x \notin b C\right\}\right] \\
& +\sum_{k=2 \delta_{0}^{-1} b \lambda \max a_{j}^{\prime}+1}^{\infty} E_{s}\left[\inf \left\{\|x\|_{1}: S_{k}+x \notin b C\right\}\right] .
\end{aligned}
$$


For some $\kappa_{1}>0$, the first term is bounded by

$$
\sum_{k=1}^{2 \delta_{0}^{-1} b \lambda \max a_{j}^{\prime}} E_{s}\left[\inf \left\{\|x\|_{1}: S_{k}+x \notin b C\right\}\right] \leq \kappa_{1} \lambda^{2} b^{2} .
$$

For $k>2 \delta_{0}^{-1} b \lambda \max a_{j}^{\prime}$,

$$
E_{s}\left[S_{k}^{\top} v_{j}^{\prime}\right] \leq s^{\top} v_{j}^{\prime}-k \delta_{0}<-k \delta_{0} / 3 .
$$

Also an estimate of the second term of (33) can be easily obtained by virtue of the fact that $X_{k}$ 's are i.i.d. multivariate regularly varying random vectors. In particular, there exists a $\kappa_{1}>0$ such that

$$
\sum_{k=2 \delta_{0}^{-1} b \lambda \max a_{j}^{\prime}+1}^{\infty} E_{s}\left[\inf \left\{\|x\|_{1}: S_{k}+x \notin b C\right\}\right] \leq \kappa_{1} b^{-\alpha+2} .
$$

Thus, the conclusion of Lemma 4 holds for $\alpha>3$.

Applying the weak convergence result in Proposition 1 together with the above lemma yields

$$
E\left[\beta(b) / b^{2} ; S_{\tau_{b A}} \in L_{\lambda}(b) \mid \tau_{b A}<T(b)\right] \rightarrow E\left[s_{\beta}(Y(Z)+\eta Z, Z) ; Y(Z)+\eta Z \in b^{-1} L_{\lambda}(b) \mid Z<T^{*}\right],
$$

and in turn, the right-hand side of asymptotic relation converges to

$$
E\left[s_{\beta}(Y(Z)+\eta Z, Z) \mid Z<T^{*}\right] \quad \text { as } \lambda \rightarrow \infty .
$$

So, it suffices to show that the second term on the right-hand side of (32) is negligible when sufficiently large $\lambda$ is chosen. First observe that

$$
E\left[\beta(b) ; S_{\tau_{b A}} \in L_{\lambda}^{c}(b) \mid \tau_{b A}<T(b)\right]=\frac{E\left[\beta(b) ; S_{\tau_{b A}} \in L_{\lambda}^{c}(b) ; \tau_{L^{c}} \leq \tau_{b A}<T(b)\right]}{P\left(\tau_{b A}<T(b)\right)}
$$

where $\tau_{L^{c}}=\inf \left\{k: S_{k} \in L_{\lambda}^{c}(b)\right\}$. The numerator on the right-hand side of the above equation is

$$
E\left[\beta(b) ; S_{\tau_{b A}} \in L_{\lambda}^{c}(b) ; \tau_{L^{c}} \leq \tau_{b A}<T(b)\right] \leq E\left[\sum_{k=\tau_{L^{c}}}^{T(b)} \inf \left\{\|x\|_{1}: S_{k}+x \notin b C\right\} ; \tau_{L^{c}}<T(b)\right] .
$$

Similar to the previous proof, we will apply Lemma 2 by constructing an appropriate Lyapunov function. For notational convenience, we still use $g_{b}(s)$ to denote the Lyapunov function but with a different definition. Let us redefine

$$
h_{b}(s)=\sum_{j=1}^{m^{\prime}}\left(\kappa b \lambda+b \lambda a_{j}^{\prime}-s^{\top} v_{j}^{\prime}\right)^{2} G_{j}\left(b \lambda a_{j}^{\prime}-s^{\top} v_{j}^{\prime}\right),
$$

where $G_{j}$ is the integrated tail as in (26). With the above $h$-function, a candidate of the Lyapunov function is defined as

$$
g_{b}(s)=\left\{\begin{array}{ll}
\min \left(\kappa_{2} h_{b}(s), m^{\prime} \kappa b^{2} \lambda^{2}\right) & \text { if } s \in L_{\lambda}(b) \\
\max \left(\kappa_{3} \max _{j}\left(s^{\top} v_{j}^{\prime}-b a_{j}^{\prime}\right)^{2}, m^{\prime} \kappa b^{2} \lambda^{2}\right) & \text { if } s \in L_{\lambda}^{c}(b)
\end{array},\right.
$$

for some $\kappa, \kappa_{2}$, and $\kappa_{3}$ (that will be chosen to be large enough). The following lemma presents the boundary condition of the $g_{b}(s)$. 
Lemma 5 By choosing $\kappa$ and $\kappa_{3}$ large, we have that

$$
g_{b}(s) \geq E_{s}\left[\sum_{k=\tau_{L^{c}}}^{T(b)} \inf \left\{\|x\|_{1}: S_{k}+x \notin b C\right\} ; \tau_{L^{c}}<T(b)\right],
$$

for all s such that $g_{b}(s) \geq m^{\prime} \kappa b^{2} \lambda^{2}$.

Again the proof of the above lemma is completely analogous to that of Lemma 4 and therefore is omitted.

For the rest of the proof, we will show that $g_{b}(s)$ solves the Lyapunov inequality so that $g_{b}(0)$ is an upper bound of (24). That is, we need to verify

$$
g_{b}(s) \geq E\left[g_{b}(s+X)\right] \quad \text { for } g_{b}(s)<m^{\prime} \kappa \lambda^{2} b^{2} .
$$

We rewrite the right-hand side of the above inequality as

$$
\begin{aligned}
& E\left[g_{b}(s+X)\right] \\
& \quad=E\left[g_{b}(s+X) ; X \notin C_{a}(s)\right]+E\left[g_{b}(s+X) ; X \in C_{a}(s)\right] \\
& \quad=J_{1}+J_{2},
\end{aligned}
$$

where

$$
C_{a}(s)=\cup_{j=1}^{m^{\prime}}\left\{y: y^{\top} v_{j}^{\prime}>a\left(b \lambda a_{j}^{\prime}-s^{\top} v_{j}^{\prime}\right)\right\} .
$$

For the $J_{2}$ term, there exists some $\kappa_{4}>0$ such that

$$
\begin{aligned}
J_{2} & =E\left[g_{b}(s+X) ; X \in C_{a}(s)\right] \\
& =E\left[g_{b}(s+X) \mid X \in C_{a}(s)\right] P\left[X \in C_{a}(s)\right] \\
& \leq \kappa_{2}\left[m^{\prime} \kappa \lambda^{2} b^{2}+\sum_{j=1}^{m^{\prime}} \kappa_{3} \zeta\left(b \lambda a_{j}^{\prime}-s^{\top} v_{j}^{\prime}\right)^{2} w_{j}\right] P\left[X \in C_{a}(s)\right] .
\end{aligned}
$$

For the $J_{1}$ term, an analogous argument works in the case of Theorem 1 . We use Rolle's representation and write $J_{1}$ as

$$
\begin{aligned}
J_{1} & =E\left[g_{b}(s+X) ; X \notin C_{a}(s)\right] \\
& \leq g_{b}(s)+E\left[X^{\top} \partial g_{b}(s+\theta X) ; X \notin C_{a}(s)\right],
\end{aligned}
$$

where $\theta \in(0,1)$ may depends on $X$. The gradient of $g_{b}$ is

$$
\begin{aligned}
& \partial g_{b}(s)= \kappa_{2} \sum_{j=1}^{m^{\prime}} v_{j}^{\prime}\left(\kappa b \lambda+b \lambda a_{j}^{\prime}-s^{\top} v_{j}^{\prime}\right) \\
& \quad \times\left[-2 G_{j}\left(b \lambda a_{j}^{\prime}-s^{\top} v_{j}^{\prime}\right)+\left(\kappa b \lambda+b \lambda a_{j}^{\prime}-s^{\top} v_{j}^{\prime}\right) P\left(X^{\top} v_{j}^{\prime}>b \lambda a_{j}^{\prime}-s^{\top} v_{j}^{\prime}\right)\right] \\
&=\kappa_{2} \sum_{j=1}^{m^{\prime}} v_{j}^{\prime}\left(\kappa b \lambda+b \lambda a_{j}^{\prime}-s^{\top} v_{j}^{\prime}\right) \\
& \quad \times\left[\kappa b \lambda P\left(X^{\top} v_{j}^{\prime}>b \lambda a_{j}^{\prime}-s^{\top} v_{j}^{\prime}\right)+\{\alpha-3+o(1)\} G_{j}\left(b \lambda a_{j}^{\prime}-s^{\top} v_{j}^{\prime}\right)\right],
\end{aligned}
$$


where the $o(1)$ is as $\min _{j}\left(b \lambda a_{j}^{\prime}-s^{\top} v_{j}^{\prime}\right) \rightarrow \infty$. For a small $\varepsilon_{0}>0$, by the dominated convergence theorem, one finds

$$
\begin{aligned}
& E\left[X^{\top} \partial g_{b}(s+\theta X) ; X \notin C_{a}(s)\right] \\
& \quad \leq-\delta_{0}\left(\kappa_{2}-\varepsilon_{0}\right) \sum_{j=1}^{m^{\prime}}\left(\kappa b \lambda+b \lambda a_{j}^{\prime}-s^{\top} v_{j}^{\prime}\right) \\
& \quad \times\left[\kappa b \lambda P\left(X^{\top} v_{j}^{\prime}>b \lambda a_{j}^{\prime}-s^{\top} v_{j}^{\prime}\right)+\{\alpha-3+o(1)\} G_{j}\left(b \lambda a_{j}^{\prime}-s^{\top} v_{j}^{\prime}\right)\right],
\end{aligned}
$$

which is sufficiently small for $\min _{j}\left(b \lambda a_{j}^{\prime}-s^{\top} v_{j}^{\prime}\right.$ ). We can choose sufficiently large $\kappa_{2}$ (depending on $\kappa_{4}$ ) such that

$$
E\left[X^{\top} \partial g_{b}(s+\theta X) ; X \notin C_{a}(s)\right]+J_{2} \leq 0,
$$

for $\min \left(b \lambda a_{j}^{\prime}-s^{\top} v_{j}^{\prime}\right)$ large enough. Together with the approximation of $P\left(\tau_{b A}<\infty\right)$, it follows that

$$
\frac{E\left[\beta(b) ; S_{\tau_{b A}} \in L_{\lambda}^{c}(b) ; \tau_{b A}<T(b)\right]}{P\left(\tau_{b A}<T(b)\right)} \leq \frac{g_{b}(0)}{\varepsilon_{0} P\left(\tau_{b A}<\infty\right)} \leq \kappa_{6} \lambda^{-\alpha+3} b^{2} .
$$

Thus, for any $\varepsilon>0$, we can choose $\lambda$ sufficiently large such that

$$
E\left[\beta(b) ; S_{\tau_{b A}} \in L_{\lambda}^{c}(b) \mid \tau_{b A}<T(b)\right]<\varepsilon b^{2} .
$$

Substitution this into (32) leads to the desired conclusion.

\section{Acknowledge}

We would like to thank the editor and the referee for their valuable comments. This research is supported in part by NSF CMMI-1069064 and SES-1123698.

\section{References}

[1] S. Asmussen and H. Albrecher. Ruin Probabilities, volume 14 of Advanced Series on Statistical Science and Applied Probability. World Scientific Singapore, 2 edition, 2010.

[2] F. Avram, Z. Palmowski, and M. Pistorius. A two-dimensional ruin problem on the positive quadrant. Insurance: Mathematics and Economics, 42(1):227-234, 2008a.

[3] A.L. Badescu, E.C.K. Cheung, and L. Rabehasaina. A two-dimensional risk model with proportional reinsurance. Journal of Applied Probability, 48:749-765, 2011.

[4] B. Basrak, R.A. Davis, and T. Mikosch. A characterization of multivariate regular variation. Annals of Applied Probability, 12(3):908-920, 2002. 
[5] R. Biard, S. Loisel, C. Macci, and N. Veraverbeke. Asymptotic behavior of the finite-time expected time-integrated negative part of some risk processes and optimal reserve allocation. Journal of Mathematical Analysis and Applications, 367:535-549, 2010.

[6] J. Blanchet and J. Liu. State-dependent importance sampling for regularly varying random walks. Advances in Applied Probability, 40(4):1104-1128, 2008.

[7] J. Blanchet and J. Liu. Total variation approximations and conditional limit theorems for multivariate regularly varying random walks conditioned on ruin. Bernoulli, to appear and available at http://www.bernoulli-society.org/index.php/publications/bernoulli-journal/bernoullijournal-papers.

[8] J. Cai and H. Li. Multivariate risk model of phase type. Insurance: Mathematics and Economics, 36(2):137-152, 2005.

[9] J. Cai and H. Li. Dependence properties and bounds for ruin probabilities in multivariate compound risk models. Journal of Multivariate Analysis, 98(4):757-773, 2007.

[10] W.-S. Chan, H. Yang, and L. Zhang. Some results on the ruin probability in a two-dimensional risk model. Insurance: Mathematics and Economics, 32(3):345-358, 2003.

[11] J.F. Collamore. Hitting probabilities and large deviations. The Annals of Probability, 24(4):2065-2078, 1996.

[12] J.F. Collamore. Importance sampling techniques for the multidimensional ruin problem for general markov additive sequences of random vectors. Annals of Applied Probability, 12(1):382$421,2002$.

[13] H. Cramér. On some questions connected with mathematical risk. University of California Publications in Statistics, 2:99-123, 1954.

[14] I. Czarna and Z. Palmowski. De finetti's dividend problem and impulse control for a twodimensional insurance risk process. Stochastic Models, 27(2):220-250, 2011.

[15] L. Dang, N. Zhu, and H. Zhang. Survival probability for a two-dimensional risk model. Insurance: Mathematics and Economics, 44(3):491-496, 2009.

[16] A. Dembo, S. Karlin, and O. Zeitouni. Large exceedances for multidimensional lévy processes. Annals of Applied Probability, 4:432-447, 1994.

[17] A.D. dos Reis. On the moments of ruin and recovery times. Insurance: Mathematics and Economics, 27(3):331-343, 2000. 
[18] H.U. Gerber, E.S.W. Shiu, and H. Yang. The omega model: from bankruptcy to occupation times in the red. European Actuarial Journal, 2(2):259-272, 2012.

[19] L. Gong, A.L. Badescu, and E.C.K. Cheung. Recursive methods for a multi-dimensional risk process with common shocks. Insurance: Mathematics and Economics, 50(1):109-120, 2012.

[20] H. Hult and F. Lindskog. Heavy-tailed insurance portfolios: buffer capital and ruin probabilities. School of ORIE, Cornell University, Technical Report, 1441, 2006.

[21] H. Hult, F. Lindskog, T. Mikosch, and G. Samordnitsky. Functional large deviations for multivariate regularly varying random walks. Annals of Applied Probability, 15:2651-2680, 2005 .

[22] J. Li, Z. Liu, and Q. Tang. On the ruin probabilities of a bidimensional perturbed risk model. Insurance: Mathematics and Economics, 41(1):185-195, 2007.

[23] S. Loisel. Differentiation of some functionals of risk processes, and optimal reserve allocation. The Journal of Applied Probability, 42(2):379-392, 2005.

[24] S.V. Nagaev. Integral limit theorems for large deviations when cramér's conditions are not fulfilled i, ii. Theory of Probability and its Application, 14:51-64, 193-208, 1969.

[25] S.V. Nagaev. Limit theorems for large deviations where cramér's conditions are violated (in russian). Izv. Akad. Nauk UzSSR Ser. Fiz.Mat. Nauk, 6:17-22, 1969.

[26] S.V. Nagaev. Large deviations of sums of independent random variables. Annals of Probability, 7:745-789, 1979.

[27] L. Rabehasaina. Risk processes with interest force in markovian environment. Stochastic Models, 25(4):580-613, 2009.

[28] S.I. Resnick. Heavy Tail Phenomena: Probabilistic and Statistical Modeling. Series in Operations Research and Financial Engineering. Springer, New York, 2006.

[29] K.C. Yuen, J. Guo, and X. Wu. On the first time of ruin in the bivariate compound poisson model. Insurance: Mathematics and Economics, 38(2):298-308, 2006. 